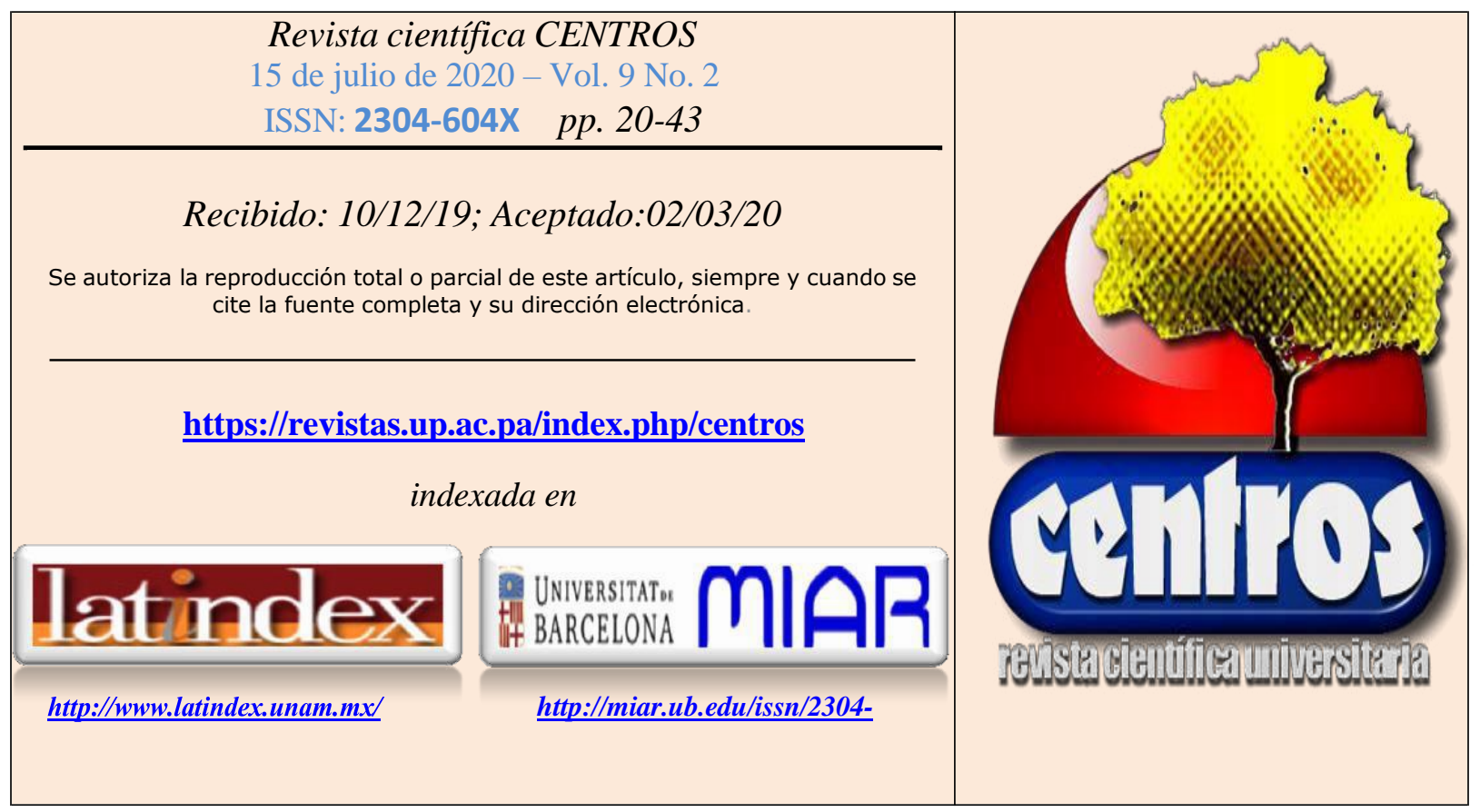

\title{
Posicionamiento Web Móvil en sitios web de empresas e instituciones panameñas con estándares internacionales
}

\section{Mobile Web Positioning on Websites of Panamanian Companies and Institutions with International Standars}

Cindy Esquivel. ${ }^{1}$; Ángel Ávila ${ }^{1}$; Eliecer Espinosa ${ }^{1}$ y Darnell Gálvez ${ }^{1}$

${ }^{1}$ Universidad De Panamá. Facultad De Informática, Electrónica y Comunicación. cindy.esquivel@up.ac.pa.

\section{Resumen}

En la actualidad, el uso del móvil para navegar en la web ha tenido un auge, importantísimo, para los usuarios y, en nuestro país, crece cada vez más. El posicionamiento en buscadores ha cambiado mucho en los últimos años. Ha pasado de un indexado que permitía que los contenidos más visibles fueran los que aprovechaban mejor el algoritmo a su favor, a una época en el que el posicionamiento responde a una obsesión por el servicio al usuario.

Es un campo que empezó desde el momento en que los motores de 
búsquedas ganaran la partida a los primeros directorios a principios de este siglo. Desde un inicio, se creó una guerra permanente entre los intereses de los buscadores (mostrar el mejor contenido) y los de los desarrolladores (posicionar su web). Conforme los buscadores, y en especial Google, fueron teniendo más datos sobre el comportamiento de los usuarios y sus búsquedas, fueron afinando más sus algoritmos. Al mismo tiempo, los SEO seguían intentando desarrollar técnicas para manipular sus resultados. Actualmente, el SEO se adapta al día a día de los usuarios para ayudarlos a relacionarse más fácilmente con el mundo, con un proceso de indexado cada vez más parecido al del criterio humano. Lo anterior, nos lleva a hacer esta investigación, cuyo objetivo es: Evaluar el posicionamiento Web Móvil en sitios web de empresas e instituciones panameñas.

Esta fue dividida en cuatro etapas. En la primera se hizo la revisión de la literatura, sobre el posicionamiento web móvil; en la segunda, la selección, evaluación y elaboración de una lista de herramientas para complementarla; en la tercera, se evaluaron los sitios seleccionados, utilizando la herramienta informática en línea; y en la cuarta se hizo la tabulación de la información obtenida. Esto dio como resultado que la accesibilidad móvil sigue creciendo en nuestro país y la optimización SEO MÓVIL es obligatoria y, en Panamá a pesar de que su desarrollo cada día es de mayor crecimiento, no todos los sitios web cuentan con una página optimizada con el SEO MÓVIL y si lo cuentan no está optimizada de una manera adecuada con los estándares necesarios para tener una de las principales posiciones de los buscadores. Con el fin de mejorar el posicionamiento Web móvil y obtener un adecuado rendimiento del sitio web es indispensable utilizar las herramientas SEO móvil, ya que son fundamentales porque ayudan a analizar el estado de las páginas web y nos brindan indicaciones acerca de lo que debemos mejorar.

Palabras Clave: Páginas Web, Estándares, Desarrolladores web, Posicionamiento Web Móvil, Evaluación. 


\section{Abstrac}

Currently the use of mobile phones to surf the web is being developed with greater importance by users and its growth is increasingly high in our country. Search engine positioning has changed a lot in recent years. It has gone from an indexing allowing the most visible contents to become the ones that took better advantage of the algorithm in their favor, in an era in which positioning responds to an obsession to provide service to the users

It is a field that started from the moment that search engines won over the first directories at the beginning of this century. From the first moment, a permanent war was created between the interests of the search engines (to show the best content) and those of the developers (to position their web). As search engines, and especially Google, got more data about user behavior and searches, they refined their algorithms. At the same time, SEOs tried to develop techniques to manipulate their results. Currently, SEO is adapted to the day-to-day life of users. The aim is to help them to relate more easily to the world, with an indexing process that is increasingly similar to that of human judgement. The aim of our research is: To evaluate the Mobile Web Positioning in Panamanian companies and Institutions' websites. For the development of this study we used the following method that consisted of four (4) stages. The first stage, literature review, about mobile web positioning. The second stage, selection, evaluation and elaboration of a list of tools.

For the third stage, the selected sites will be evaluated, using the online computer tool The fourth stage is the tabulation of the information obtained. As a result, the mobile accessibility continues to grow in our country and the SEO MOBILE is mandatory and in Panama, despite the fact that its development is growing every day, not all websites have a page optimized with the Seo Mobile and if they do, it is not optimized in an adequate way with the necessary standards to have one of the main positions in the search engines. In order to improve the 
mobile web positioning and obtain an adequate performance of the website, it is essential to use the mobile SEO tools, since they are fundamental because they help to analyze the state of the web pages and to provide indications about what we should improve.

Keywords: Websites, Standards, Web Developers, Mobile Web Positioning, Evaluation.

\section{Introducción}

EI SEO nació a partir de 1993 con la aparición de las primeras páginas web, la primera apareció en 1991. En ese momento, empezaron a hacerse necesarias plataformas que catalogasen la información que contenían las páginas web para poder encontrarlas con facilidad, como pasaba con el catálogo interno de una biblioteca (Argiz, 2017).

Los administradores de páginas web y proveedores de contenido empezaron a optimizar sus sitios web para aparecer en los ránkings. Yahoo ${ }^{1}$ que entró en el año 1994, el mismo les permitía a los sitios web solicitar su indexación en su buscador, que hacía de forma manual. Con la aparición de otros buscadores como Altavista $^{2}$ y Google ${ }^{3}$ en 1995 y 1997 respectivamente, los buscadores sofisticaron todavía más la recopilación de datos de las diferentes webs para clasificarlas en sus ránkings.

Algunos de los indicadores que usaban los buscadores para clasificar las webs entonces eran: número de visitas, número de repeticiones de las palabras clave que los usuarios buscaban en los contenidos de la web, y Backlinks, es

\footnotetext{
${ }^{1}$ Yahoo: Es una empresa global de medios con sede en Estados Unidos que posee un portal de Internet, un directorio web y una serie de servicios tales como el popular correo electrónico Yahoo!

${ }^{2}$ AltaVista: Se refiere a un motor de búsqueda de Internet desarrollado por Digital Equipment Corporation.

${ }^{3}$ Google: Es una empresa multinacional estadounidense especializada en servicios y productos relacionados con software, Internet, dispositivos electrónicos y otras tecnologías.
} 
decir, número de webs externas que referenciaban esa página con links a ella.

Con la tecnología disponible en aquel momento, la manera de saber si una web coincidía con lo que el usuario buscaba era que esas palabras se repitieran, y de entre todas las webs que eran concordantes, la única manera que había de ordenarlas en el ránking ${ }^{4}$ por autoridad era medir las visitas que obtenía y que otras webs la referenciasen, de esa forma, en ese momento era habitual que una web que cumpliera esos requisitos subiera en seguida de posiciones en buscadores.

Esto fue aprovechado por empresas que empezaron a ver a los usuarios como consumidores y potenciales clientes, y querían que sus contenidos aparecieran en estos buscadores por encima de su competencia cuando los usuarios hacían búsquedas relacionadas con los productos o servicios que ofrecían. Fue este, hacia los inicios de los años 2000, el momento en el que nació el SEO como hoy lo conocemos, como el aprovechamiento del algoritmo de los buscadores con fines comerciales.

EI SEO como lo conocemos hoy en día nace de la conceptuación de los usuarios que buscan información en buscadores como consumidores: las empresas empezaron a hacer esfuerzos por usar los algoritmos de búsqueda a su favor para ofrecer productos y servicios. La optimización pasó de ser cosa de programadores a ser cosa de expertos en márketing (SEO, 2014).

En la actualidad, las búsquedas desde móvil empezaron a ganar protagonismo y el contenido relevante y accesible para móviles (como el contenido responsive ${ }^{5}$ fácilmente consultable desde smartphones ${ }^{6} \mathrm{y}$ el que tiene

\footnotetext{
${ }^{4}$ Ranking: se la usa para referir a aquel o aquello que se encuentra en una posición superior a otro.

${ }^{5}$ Responsive: Es una técnica de diseño web que busca la correcta visualización de una misma página en distintos dispositivos
}

${ }^{6}$ Smartphones: Es el término en inglés que se utiliza para denominar a un teléfono inteligente, es un equipo
celular con funciones más avanzadas que las de un teléfono corriente. 
en cuenta el tipo de público que hace una búsqueda y su intención) es especialmente valorado.

El 21 de abril, el algoritmo móvil de Google empezó hacer unos de los más importantes en la actualidad en todo el mundo, los sitios Web ahora deben ser más amigables para móviles, lo que se estima que afectará en gran medida los resultados de las búsquedas específicas para estos dispositivos, ya que cada vez más personas usan sus teléfonos inteligentes para navegar en Internet, por eso Google hizo estos cambios, para mostrar resultados más relevantes, bien sea en sitios Web amigables o en apps.

El Posicionamiento Web Móvil, cambiará nuestra vida, porque podemos encontrar tiendas, restaurantes, libros y toda la información solo haciendo una búsqueda desde nuestros móviles, mientras estamos en cualquier lugar, solo nos tomaría unos segundos. Por esta misma razón es que la mayoría de las búsquedas que se harán en Google, se realicen a través de dispositivos móviles. (Cano, 2014)

En Panamá, el uso de los móviles cada día se utiliza con mayor frecuencia en los usuarios para acceder a un sitio Web, lo cual hace que el tráfico desde terminales móviles sea mucho mayor que si acceden en una computadora de escritorio o portátil.

Según un estudio realizado por Google el $56 \%$ de las búsquedas en Panamá hasta el 2015, se realizaron a través de dispositivos móviles. (Fuenmayor 2016).

Por otro lado, el medio digital Panamá On, indica que hasta finales del 2016 la penetración de móviles es de un 170\%. (Reyes 2016). 
Estas cifras hacen de Panamá un mercado que sigue creciendo en la tecnología con usuarios que nacen y crecen dentro del consumo móvil, pues el mismo ofrece una amplia gama de oportunidades para interactuar con los usuarios a nivel más personal, lo que le da la posibilidad de llegar al público objetivo.

Es por esta razón que en este trabajo nos enfocaremos en las empresas que están incursionando en el comercio electrónico en la ciudad de Panamá, ya que para nuestro concepto son las que más necesitan contar con un Sitio Web Optimizado para móviles, porque hoy en día los usuarios realizan muchas compras por internet y es fundamental poder lograr que las mismas se puedan efectuar sin problemas en cualquier momento y en cualquier lugar, para que tengan la presencia adecuada y puedan implementar esta tendencia desde el punto de vista de Google como desde el punto de vista de la usabilidad7, y así poder brindar una excelente, accesible y rápida navegación; mejor visibilidad para los motores de búsqueda e incrementar el tráfico de su sitio web todo el tiempo.

Todo esto lo lograremos implementando diferentes herramientas que permitirán realizar un análisis de los diferentes sitios Web de comercio electrónico en la ciudad de Panamá para determinar si están optimizadas con el SEO MÓVIL y así, finalmente, brindar recomendaciones basadas en las normas de la W3C8 . (Digital, 2015).

\footnotetext{
${ }^{7}$ La usabilidad: es un término usado en diseño de sitios web que describe cómo de bien los visitantes pueden utilizar un sitio Web. Una buena usabilidad requiere que el sitio sea fácil de navegar, tenga un diseño decente, sea coherente en todas las páginas y que también sea informativo y útil para el visitante.
}

${ }^{8}$ W3C: son las siglas de World Wide Web Consortium, un consorcio fundado en 1994 para dirigir a la Web hacia su pleno potencial mediante el desarrollo de protocolos comunes que promuevan su evolución y aseguren su interoperabilidad. 
El uso de móviles ha aumentado en los últimos años. Esta modalidad ha influido en que el diseño Web responsivo sea una obligación para las páginas web, debido a que las mismas deben adaptarse a los diversos tipos de pantallas móviles existentes.

Al adaptar tu página web a las diversas pantallas móviles, te mantienes a la vanguardia, mejoras la visibilidad para los motores de búsqueda, y lo más importante mejoras la experiencia del usuario.

Los usuarios utilizan cada día más los dispositivos móviles para navegar en la web, buscar información y realizar compras. (Teresa, 2015).

Según un reciente estudio de Google, el 52\% de los usuarios indicaron que son menos propensos a involucrarse con una empresa si su página web no está optimizada para móviles.

Por eso, hoy es muy importante que las empresas tengan diseño web responsivo, para poder brindar a sus clientes mejores condiciones de navegación, y aumentar la conversión de usuarios a clientes.

También es importante señalar que el $56 \%$ de las búsquedas en Google en Panamá a noviembre del 2015 se realizaron a través de dispositivos móviles.

Estas cifras hacen de Panamá un mercado súper interesante, porque en todos los países centroamericanos hay un gran porcentaje de consumo de Internet a través de celulares; pero Panamá los sobrepasa.

El gerente general de Google para la región asegura que Panamá es un mercado que sigue creciendo con usuarios que nacen y crecen dentro del 
consumo móvil y que quizás, a la fecha, nunca han tenido una experiencia en computadoras de escritorio y es precisamente por eso que resulta importante que todas las empresas tengan la presencia adecuada en sistemas móviles, para capturar todo el tráfico, todo el tiempo y todo el interés de los usuarios. (Fuenmayor., 2016).

Panamá está en una situación única, ya que figura un alto porcentaje de consumo de Internet a través de los dispositivos móviles, lo que hace que necesitemos que los sitios web estén a la vanguardia de los requerimientos necesarios para una adecuada optimización SEO MÓVIL.

\section{Materiales Y Métodos}

En este proyecto acerca del posicionamiento Web móvil en Panamá analizamos el desarrollo de este en los sitios Web de comercio electrónico, para lo cual tomamos 50 sitios web de instituciones públicas y privadas, universidades, restaurantes y bancos.

Partiendo del proceso de identificación a través de las investigaciones y encuestas realizadas para determinar la situación actual del nivel de desarrollo del SEO MÓVIL en Panamá, llevamos a cabo un estudio que consiste en analizar los diferentes sitios web de las empresas más conocidas a nivel del comercio electrónico en nuestro país, para conocer cuáles de ellas contaban con un sitio optimizado para móviles.

Para ello, usamos varias herramientas SEO MÓVIL que facilitarían la labor, como la aplicación MOBILE-FRIENDLY TEST, PAGESPEED INSIGHTS y WEBSITE GRADER. (Lázaro, 2015).

El desarrollo de este estudio lo llevamos a cabo en cuatro etapas. La primera etapa se refiere a la revisión de la literatura, sobre el posicionamiento web móvil y los estándares existentes relacionados con las páginas y los sitios 
web. La segunda etapa, selección, evaluación y elaboración de una lista de herramientas libres disponibles en la web, basados en estándares internacionales, que serán utilizados para la evaluación técnica de las páginas y sitios web.

La evaluación se realizará en base a la disponibilidad, velocidad de respuesta, tipo respuesta proporcionada por la herramienta, incorporación de las Pautas de Accesibilidad al Contenido Web, entre otras características. (Ver cuadro 1). Para la tercera etapa se evaluarán los sitios seleccionados, utilizando la herramienta informática en línea. La cuarta etapa es la tabulación de la información obtenida, procediendo al análisis estadístico de los datos y el resumen de las tendencias encontradas en los sitios.

\section{Cuadro 1 Pautas de Accesibilidad en la Web}

\begin{tabular}{|c|c|c|c|c|}
\hline \multirow[b]{2}{*}{ Principio } & \multirow[b]{2}{*}{ Pauta } & \multicolumn{3}{|c|}{ Niveles de Conformidad } \\
\hline & & A & AA & AAA \\
\hline \multirow[t]{5}{*}{ Perceptible } & Texto alternativo & 1 & 0 & 0 \\
\hline & Sincronizable en el tiempo & 3 & 2 & 4 \\
\hline & Adaptable sin perder contenido & 3 & 0 & 0 \\
\hline & Distinguible & 2 & 3 & 4 \\
\hline & Total Perceptible & 9 & 5 & 8 \\
\hline \multirow{5}{*}{ Operable } & Accesible por teclado & 2 & 0 & 1 \\
\hline & Tiempo suficiente & 2 & 0 & 3 \\
\hline & Destellos & 1 & 0 & 1 \\
\hline & Navegable & 4 & 3 & 3 \\
\hline & Total Operable & 9 & 3 & 8 \\
\hline \multirow[t]{4}{*}{ Comprensible } & Legible entendible & 1 & 1 & 4 \\
\hline & Operación predecible & 2 & 2 & 1 \\
\hline & Ayuda en la entrada de datos & 2 & 2 & 2 \\
\hline & Total Comprensible & 5 & 5 & 7 \\
\hline \multirow[t]{2}{*}{ Robusto } & Compatibilidad & 1 & 0 & 0 \\
\hline & Total Robusto & 1 & 0 & 0 \\
\hline
\end{tabular}


Se utilizarán para el análisis las herramientas: MOBILE-FRIENDLY TEST, PAGESPEED INSIGHTS, WEBSITE.GRADER y SEO. Al final del informe encontraremos recomendaciones para mejorar el rendimiento del sitio web y la calificación.

\section{Composición de la muestra}

La composición de la muestra fue seleccionada utilizando el muestreo aleatorio simple, la muestra, se elaboró de una lista garantizando sitios que mostraban un funcionamiento óptimo. (Ver figura 2). Se consideraron 46 sitios web: 14 (28\%) correspondían a instituciones públicas y 32 (72\%) a empresas privadas, distribuidas según la actividad que desarrollada. Las de mayor representatividad fueron: instituciones del gobierno 8 (18\%), universidades 7 (16\%), ventas 7 (16\%), banca y medios y de comunicación $5(11 \%)$. El resto correspondía a Servicios, Tecnología, restaurantes, hospitales y agencias de seguros, cuya representación es de 4 al 9 por ciento. (Ver figura 3).

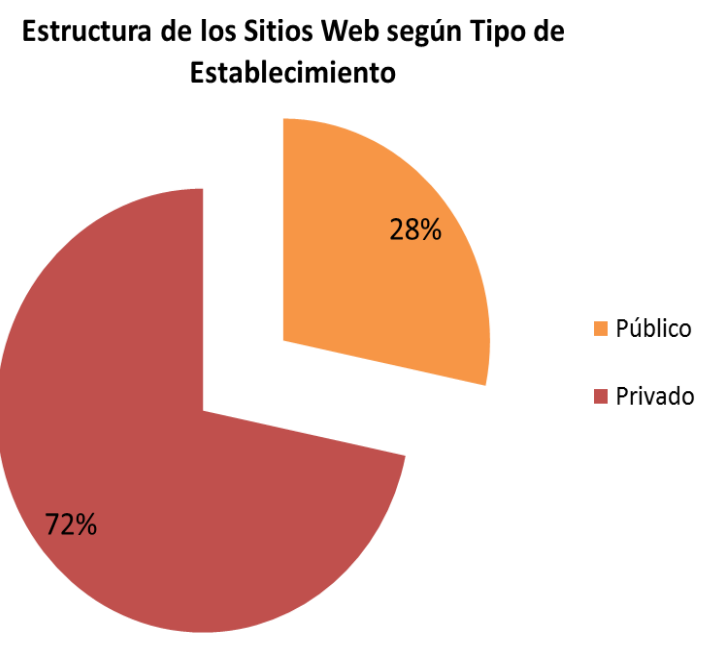

Figura 2. Estructura de la Muestra de sitios

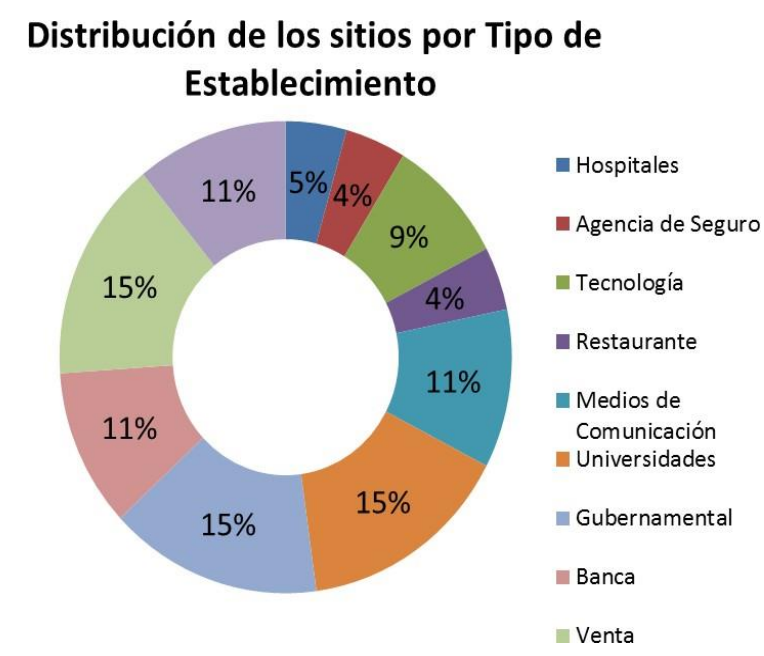

Figura 3. Distribución de los Sitios Web Panameños por tipo de Institución, según tipo de establecimiento

Los criterios estadísticos que se utilizarán para recopilar y analizar la información comprenderán dos aspectos: revisión de los resultados provenientes de los analizadores y el tipo de los establecimientos que representan los dueños de los sitios. En el cuadro 3 se observa la distribución de los sitios según tipo de institución y la actividad que desarrolla el establecimiento. ( (Cindy Esquivel, 2018). 


\section{Cuadro 3. Distribución de los Sitios Web Panameños por tipo de Institución, según tipo de establecimiento}

\begin{tabular}{|c|c|c|c|}
\hline \multirow{2}{*}{ Establecimiento } & \multicolumn{3}{|c|}{ Tipo de Institución } \\
\hline & Total & Publico & Privado \\
\hline Total & 46 & 13 & 33 \\
\hline Hospitales & 2 & O & 2 \\
\hline Agencia de Seguro & 2 & O & 2 \\
\hline Tecnología & 4 & O & 4 \\
\hline Restaurante & 2 & $\mathrm{O}$ & 2 \\
\hline Medios de Comunicación & 5 & O & 5 \\
\hline Universidades & 7 & 4 & 3 \\
\hline Gubernamental & 7 & 7 & O \\
\hline Banca & 5 & 1 & 4 \\
\hline Venta & 7 & O & 7 \\
\hline Servicio & 5 & 1 & 4 \\
\hline
\end{tabular}

Fuente: Creadas por el equipo de investigación.

\section{Resultados Y Discusión}

Estos datos nos muestran la necesidad de que las páginas web se desarrollen también en una correcta versión responsive, 100\% optimizada.

- Los smartphones tienen una penetración en la población panameña del $63 \%$.

- $\quad$ El 59\% accede a Internet desde su Smartphone cada día.

- $\quad$ El $21 \%$ de los propietarios de móviles compran a través del móvil.

- $\quad$ El $71 \%$ no sale de casa sin su dispositivo.

El fuerte impacto que tienen los teléfonos inteligentes y las "tablets" en la vida diaria de las personas cada día se vuelve más importante. Los motores de búsqueda ${ }^{9}$, nada ajenos a la realidad actual, premian las páginas web que están optimizadas para los dispositivos móviles. Además, hay tecnologías como la web responsive o como el $\mathrm{AMP}^{10}$ que permiten diseñar los sitios web de forma específica para estos dispositivos. Google y los principales buscadores son conscientes de que la inmensa mayoría de búsquedas se realizan desde los

\footnotetext{
${ }^{9}$ Motores de búsqueda: es un sistema informático que busca archivos almacenados en servidores web gracias a su spider o Web crawler.

${ }^{10}$ AMP: son las siglas de Accelerated Mobile Pages, un proyecto implementado por Google y otros socios con el objetivo de mejorar la velocidad de carga de los sitios web en los smartphones.
} 
teléfonos inteligentes y similares. Así que no queda opción si queremos que nuestro sitio web tenga un buen posicionamiento orgánico. (Baños, 2017).

Desde el año 2011, Google dispone de un robot denominado GooglebotMobile, cuya misión es rastrear contenido específico para Smartphones. Los administradores de páginas web encuentran solución a problemas y pueden revisar su funcionamiento y mejorar su rendimiento. Para ello, se puede recurrir a la "prueba de optimización para móviles".

La optimización en motores de búsqueda en español, una disciplina del marketing online que se ocupa de todas las medidas que ayudan a mejorar el posicionamiento de las páginas web en las listas de resultados de los motores de búsqueda. Asimismo, la optimización móvil (SEO para dispositivos móviles) es una parte esencial de la planificación de campañas de SEO. (Solis, 2011).

Lo importante del Seo móvil no solo tiene que ver con que las búsquedas se efectúen desde un teléfono móvil. También es importante, porque el tráfico web es bastante diferente cuando procede desde un dispositivo de estas características. Una de las más notorias es la geolocalización, que desde los teléfonos móviles es más evidente todavía. Por ejemplo, si un usuario busca los restaurantes más cercanos, la ubicación que se obtiene debe ser muy precisa para ofrecer los mejores resultados posibles.

Otro detalle importante es que los teléfonos móviles los suele utilizar una sola persona, a diferencia de las Computadoras de escritorio, que son usados por varias personas de forma muy habitual. Esto permite obtener datos mucho más precisos del historial de búsquedas, pues nos podemos hacer una idea mucho más clara de sus gustos, aficiones e intereses. (Posicionamiento Web, 2016).

También hay que tener en cuenta las características de los terminales, que 
siempre son más reducidos que una Computadora tradicional. Por ejemplo, siempre es conveniente minimizar el uso del teclado. Aunque los móviles han mejorado mucho en los últimos tiempos, lo cierto es que sigue siendo difícil teclear muchos datos. Por eso, hay que buscar la forma de que los usuarios tengan que escribir lo menos posible.

Una de las cosas más importantes en las webs móviles es que se carguen de forma rápida. La lentitud es muy penalizada por los buscadores y por los usuarios, así que todos los contenidos, gráficos e imágenes deben estar optimizados para lo que la gente espera.

También es importante señalar que el uso de los móviles no va a disminuir. Al contrario, cada vez será más frecuente acceder a internet con este tipo de dispositivos. Por esa razón debemos redoblar todos los esfuerzos para conseguir que nuestra web esté perfectamente construida para que tenga un excelente posicionamiento SEO MÓVIL (Tejada, 2016).

Por esta razón, en este trabajo nos enfocaremos en las empresas que están incursionando en el comercio electrónico en la ciudad de Panamá, ya que para nuestro concepto son las que más necesitan contar con un sitio Web Optimizado para móviles, porque hoy en día el comercio electrónico en Panamá se encuentra en el primer lugar de canales de mayor crecimiento en el país, seguido por las tiendas de conveniencia que ocupan el segundo lugar. Esto se debe, entre otras cosas, a la penetración de los smartphones en el mercado (100\% de penetración), ya que en la actualidad es muy raro encontrar personas que no usan un smartphone como medio de comunicación y esto les permite tener acceso rápido $e$ inmediato a sitios de compras por internet $u$ "online". Además de las compras de accesorios o artículos personales, el comercio "online" también permite el pago de servicios y de otras compras tan básicas como puede ser un boleto del cine, comida rápida o hasta un servicio de taxi y es fundamental poder contar con un sitio optimizado para móviles para que las mismas se puedan efectuar sin problemas en cualquier momento y en 
cualquier lugar, para que tengan la presencia adecuada y puedan implementar esta tendencia desde el punto de vista de Google como desde el punto de vista de la usabilidad, y así poder brindar:

- Una excelente, accesible y rápida navegación.

- Mejor visibilidad para los motores de búsqueda.

- Incrementar el tráfico de su sitio web todo el tiempo. (Nubeser, 2017).

Análisis del posicionamiento Web móvil en Panamá:

El número de usuarios de teléfonos inteligentes está creciendo continuamente y supera por mucho los usuarios de computadora. Esa es la razón por la que la posibilidad de llegar a la audiencia por este medio no debe ser ignorada.

La accesibilidad móvil sigue creciendo en nuestro país y la optimización SEO MÓVIL es obligatoria y en Panamá a pesar de que su desarrollo cada día es de mayor crecimiento no todos los sitios web cuentan con una página optimizada con el SEO Móvil y si lo cuentan no está optimizada de una manera adecuada con los estándares necesarios para tener una de las principales posiciones de los buscadores y por tal razón, mediante las herramientas web que utilizamos brindarán un análisis exhaustivo de los principales sitios del comercio electrónico en donde evaluarán cuáles de ellas cuentan con un buen posicionamiento Web móvil y cuáles necesitan adecuarse a esta tendencia que está revolucionando Panamá y así no solamente ser un país desarrollado y actualizado en el mercado móvil, sino también con los mejores sitios del comercio electrónico optimizado para móviles para lograr las mejores posiciones en los buscadores. (Digital, 2015). 


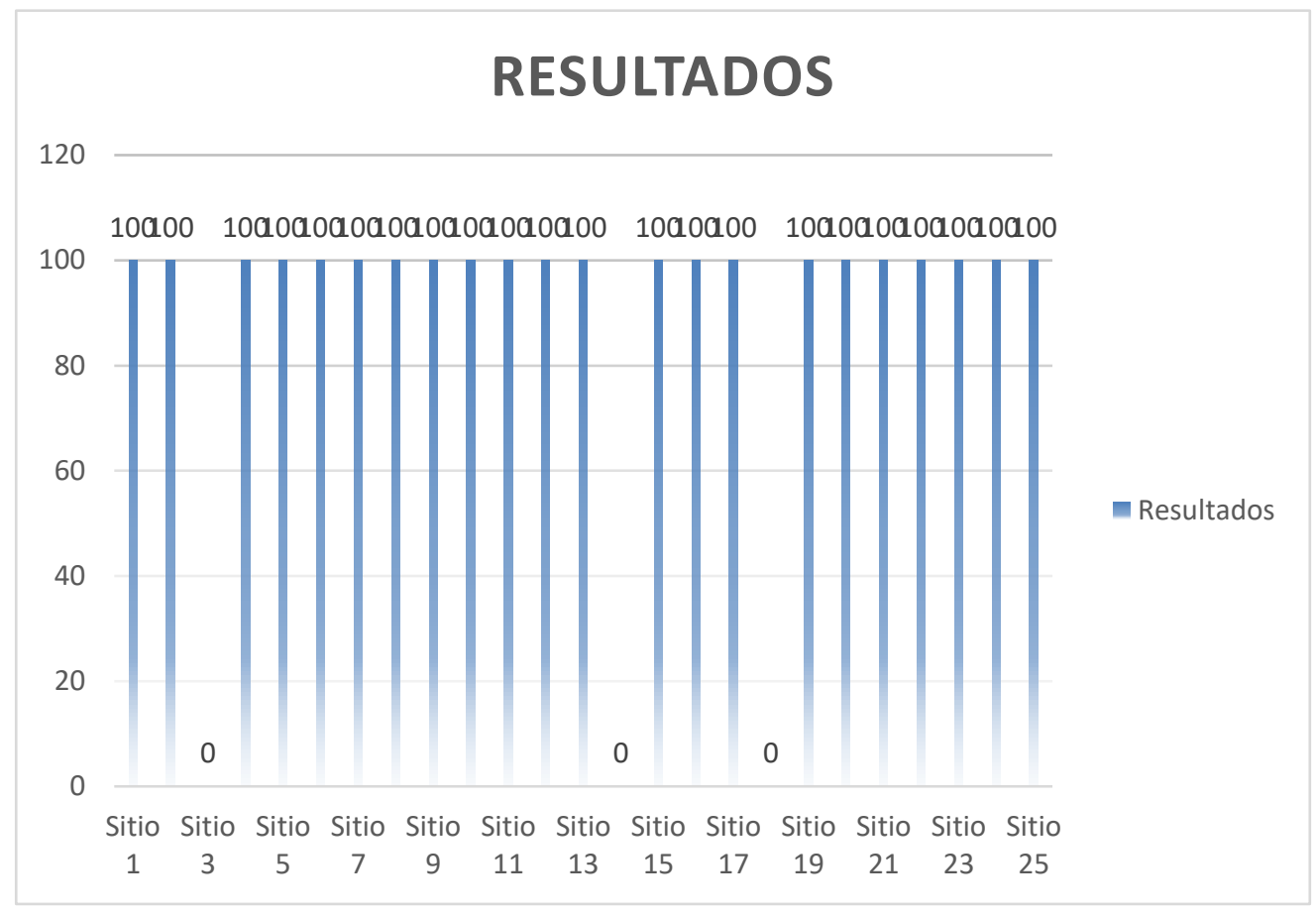

Figura 1 Gráfica Elaborada de los resultados de cada Sitio con la Herramienta Mobile-Friendly Test 


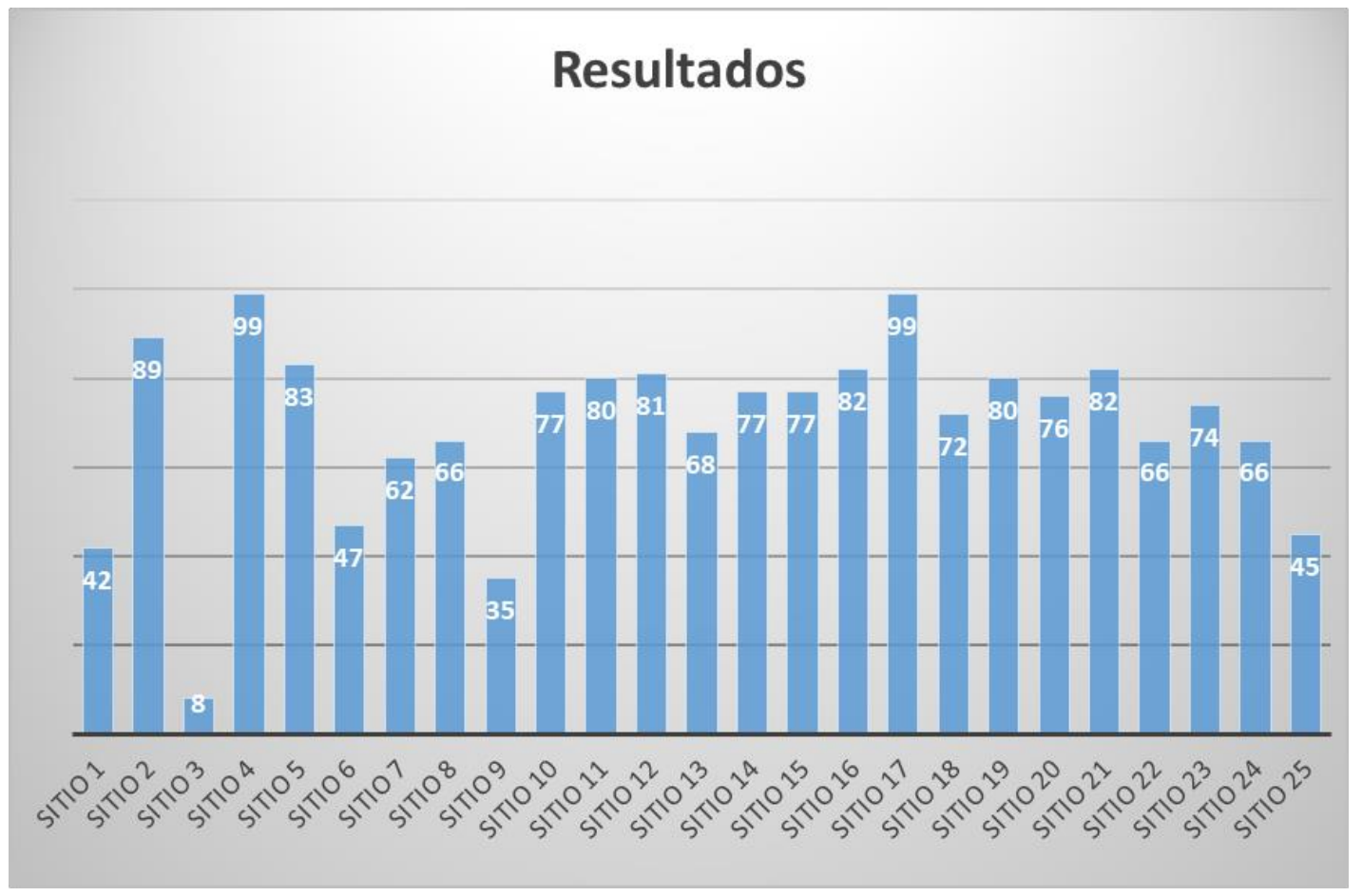

Figura 2 Gráfica Elaborada de los resultados de cada Sitio con la Herramienta Page Speed Insights

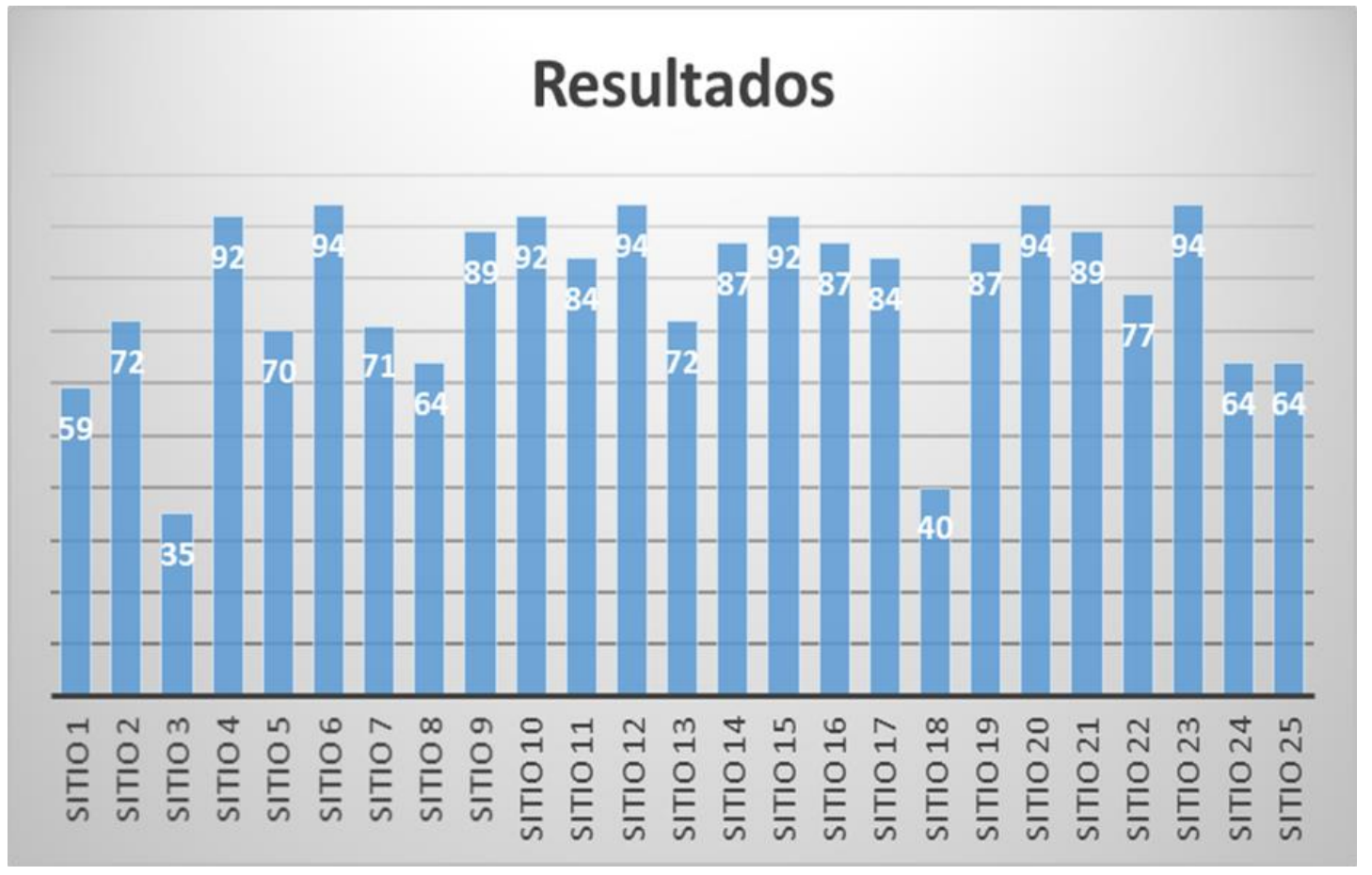

Figura 3 Gráfica Elaborada de los resultados de cada Sitio con la Herramienta Website Grader 


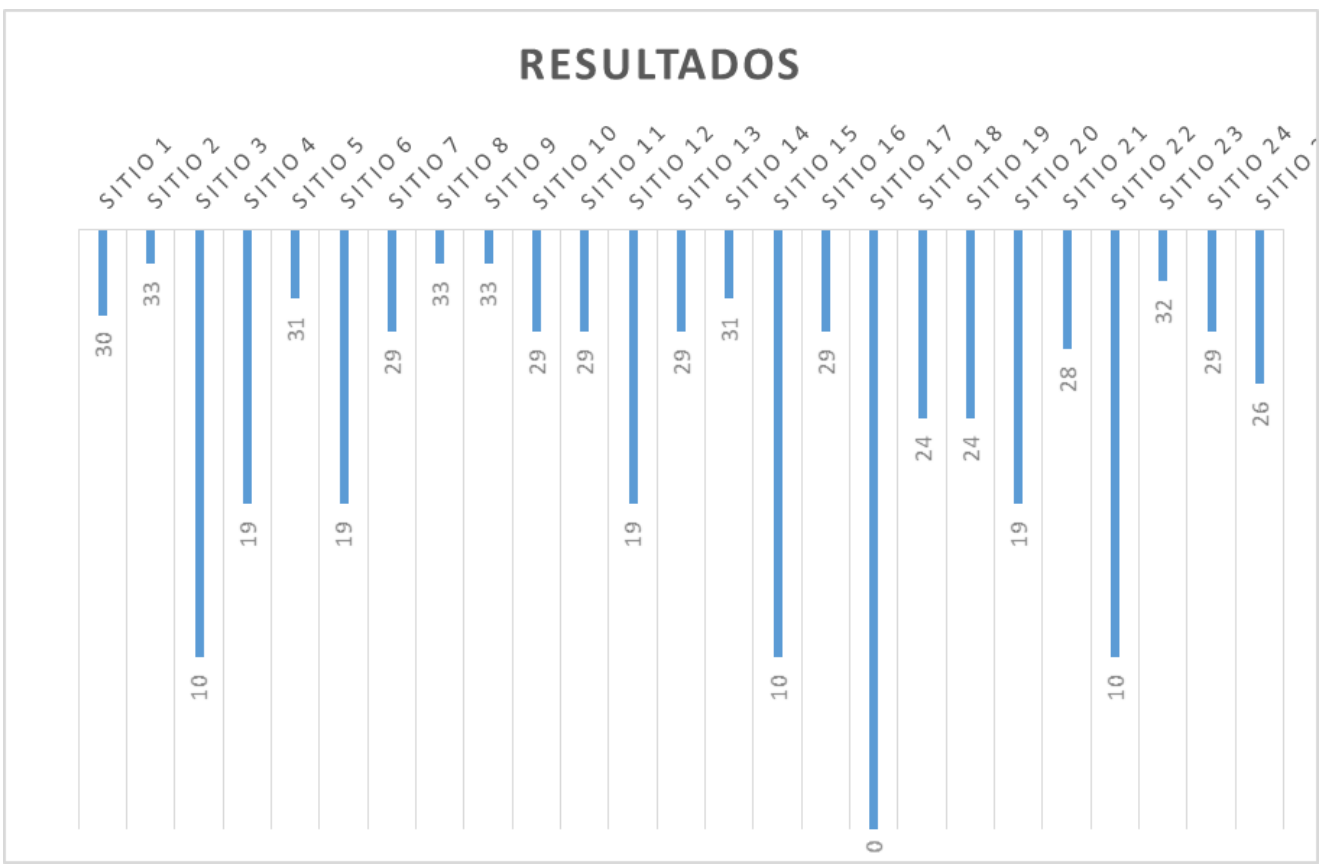

Figura 4 Gráfica Elaborada de los resultados de cada Sitio con la Herramienta Prueba mi Sitio

\section{Conclusiones}

Se puede concluir que los usuarios llevan consigo el móvil, estén donde estén, convirtiéndose en una extensión suya personal y prefieren navegar en la web desde sus teléfonos móviles para poder consultar en cualquier momento y lugar el contenido que precisen.

Por tal razón, es fundamental brindarle al usuario un adecuado sitio que se pueda mostrar en su celular sin problemas. Uno de los objetivos de esta investigación es dar a conocer la importancia de la optimización a través de los móviles a los sitios web como a los usuarios.

El posicionamiento web móvil ayuda a los sitios web a poder estar en las primeras filas de los buscadores. Esto brinda un gran beneficio ya que el sitio se da a conocer permitiendo aumentar significativamente la popularidad, facilitando que los motores de búsqueda encuentren la página y, a su vez, le permita al 
usuario sugerir el portal a otras personas.

Con el fin de mejorar el posicionamiento Web móvil y obtener un adecuado rendimiento del sitio web es indispensable utilizar las herramientas SEO M+OVIL, ya que son fundamentales porque ayudan a analizar el estado de las páginas web y nos brindan indicaciones acerca de lo que debemos mejorar.

\section{Referencias Bibliográficas}

Acosta, T., \& Luján-Mora, S. (2017). Análisis de la accesibilidad de los sitios web de las universidades ecuatorianas de excelencia. Enfoque UTE, 8(1), pp46.

http://ingenieria.ute.edu.ec/enfoqueute/index.php/revista/article/view/133

Agencia, E. d. (2014). Aplicaciones Informáticas para personas con Discapacidad.

Requisitos de Accesibilidad para Contenidos Web. Madrid: AENOR.

AL_Dwairi, K. M., Faba-Pérez, C., \& Vargas-Quesada, B. (30 de noviembre de 2010). Indicadores Webmétricos de carácter formal para evaluar el posicionamiento de las universidades: el caso de los Países Árabes. INVESTIGACIÓN BIBLIOTECOLÓGICA,145-171.Obtenido dehttp://www.ugr.es/ benjamin/Indicadores-webmetricos.pdf Alicante, U. d. (s.f.). Pautas de accesibilidad al Contenido Web 2.0. Recuperado el octubre de 2014, de http://accesibilidadweb.dlsi.ua.es/?menu=niveles-2.0

Álvarez-Álvarez, C. (2017). ¿Qué me ofrecen las páginas web de los centros educativos? Estudio exploratorio en Cantabria (España).

REICE. Revista Iberoamericana sobre Calidad, Eficacia y Cambio en Educación. Tomado de (Argiz, 2017) (Argiz, 2017)A. N. (2011). Estándares para las Páginas Web en las Entidades del Gobierno. Panamá: Gaceta Oficial Digital. 
Argiz, P. C. (26 de enero de 2017). Historia del SEO y la evolución que ayuda a entenderlo hoy en día. Obtenido de SiteLabs: https://sitelabs.es/historiadel-seo-evolucion/

Arias, A. (18 de mayo de 2016). Davilac. Obtenido de Davilac: https://www.davilac.net/1545/mobile/mobile-friendly-test-nuevaherramienta-de-google/

Balsells, L. A. C., González, J. C. G., Balsells, M. A. C., \& Chamorro, V. A. P. (2017). La accesibilidad de los portales web de las universidades públicas andaluzas. Revista española de Documentación Científica, 40(2), $169 . \quad$ Tomado de http://redc.revistas.csic.es/index.php/redc/article/view/973

Baños, J. C. (23 de enero de 2017). frikipandi. Obtenido de frikipandi: https://www.frikipandi.com/internet/20170116/google-recomiendaoptimizar-las-web-mobiles/

Barrera, C. R., Núñez Amaro, S., \& Motola Pedroso, D. (2006). Evaluación de sitios Web en Internet: Propuestas para la evaluación de sitios web de bibliotecas públicas y de salud. Acimed, 14(4), Tomado de http://scielo.sld.cu/scielo.php?pid=S1024352006000400004\&script=s ci_arttext\&tl ng=en

Bustamante, A. (20 de 05 de 2013). Cuestionan alto costo para rediseñar el sitio web de la Defensoría del Pueblo.

Obtenido de La Prensa:

http://www.prensa.com/uhora/locales/cuestionan-costo-portal-webdefensoria- pueblo-panama-contrato-directo-cd/178831

Cano, E. (24 de enero de 2014). iebschool. Obtenido de iebschool: https://www.iebschool.com/blog/desarrollo-seo-moviles-mobile marketing/ 
Carvajal, M., \& Saab, J. (23 de agosto de 2010). Lineamientos y metodologías en Usabilidad del Gobierno en Línea. Manual para la implementación del decreto 1151 Obtenido de http://paginasweb.univalle.edu.co/reglamentos/pasos/documentos/GE L108_CINT EL_Lineamientos_y_metodologias_en_usabilidad.pdf

Cindy Esquivel, D. R. (2018). Cumplientos de sitios web con los estandares internacionales. Revista Centros, 1-20.

Codina, L. (5 de febrero de 2017). Medium. Obtenido de Medium: https://medium.com/@lcodina/an\%C3\%A1lisis-de-medios-decomunicaci\%C3\%B3n-con-website-grader-5b299133a82c

Claros, I. D., \& Collazos, C. A. (Nov de 2006). Propuesta Metodológica para la Evaluación de la Usabilidad en Sitios Web: Experiencia Colombiana. Recuperado el Junio de 2014, de http://aipo.es/articulos/4/18.pdf

Comunicación, C. T. ( 2008). TAW. Recuperado el mayo de 2014, de http://www.tawdis.net/

Correa, N. (2014). Criterios para evaluar la calidad de un sitio web. Serie Competencias informacionales e informáticas(4), 1-4.

Delgado, I. H. (27 de julio de 2018). diseno web akus. Obtenido de diseno web akus: https://disenowebakus.net/estandares-web.php

Departamento de Pedagogía Aplicada 08193 Bellaterra (Barcelona). España.

Tomado de: Gubernamental Hassan, Yusef and Martín Fernández, Francisco

J. and lazza, Ghzala Diseño Web Centrado en el Usuario: Usabilidad y Arquitectura de la Información. Hipertext.net, 2004, n.2

Tomado de http://eprints.rclis.org/8998/1/Dise\%C3\%B10_Web_Centrado_en_el_ Usuario_Us abilidad_y_Arquitectura_de_la_Informaci\%C3\%B3n.pdf 
Digital, M. (28 de septiembre de 2015). Mas Digital. Obtenido de Mas Digital: https://www.masdigital.net/nuestro-blog/seo-en-panama

FDLC, F. p. (2008). Resumen Ejecutivo: Índice de Integridad 2008 de las Instituciones Publicas de Panamá. Resumen Ejecutivo, Capítulo Panameño de Transparencia Internacional, Panamá.

Fuenmayor., S. (4 de abril de 2016). El capital financiero. Obtenido de El capital financiero: https://elcapitalfinanciero.com/google-apuesta-por-eldesarrollo-movil-de-panama/

Graells, P. M. (1999). Criterios para la clasificación y evaluación de espacios web de interés educativo. Educar, (25), 95-111. Universitat Autònoma de Barcelona.

Lázaro, M. (11 de mayo de 2015). Hablando en Corto. Obtenido de Hablando en Corto: https://www.hablandoencorto.com/2015/05/herramientasoptimizacion-movil.html

Mendoza, E. (25 de 08 de 2013 ). Señalan errores en sitio web. Obtenido de La Prensa: http://www.prensa.com/impreso/panorama/senalanerrores-sitio-web/201020

Nubeser. (21 de septiembre de 2017). Nubeser. Obtenido de Nubeser: https://nubeser.com/importancia-seo-movil-seo-para-smartphone/

Oficial, G. (2002). Ley 6. Ley, Asamblea Legislativa. Obtenido de Ley $N^{\circ} 6$ : http://www.legalinfopanama.com/legislacion/administrativo/00195.pdf

Posicionamiento Web. (26 de mayo de 2016). Obtenido de Posicionamiento Web: http://posicionamientoweb.com/blog/importancia-seo-moviles/ Ramos V., Irene y Clabo, Néstor. (2008) Calidad de las sedes web de las OTRI universitarias andaluzas: contenidos, usabilidad y accesibilidad. Revista española de documentación Científica, Vol 31, No 3. Tomado de: http://redc.revistas.csic.es/index.php/redc/article/view/434

Robert B., C., Nuñez Amaro, S., \& Motola R., D. (2006). Evaluación de sitios 
Web en Internet. Propuestas para la evaluación de sitios web de bibliotecas públicas y de salud. Acimed, 14(4 http://bvs.sld.cu/revistas/aci/vol14_4_06/aci04406.htm).

Sampieri, R. (. (2006). Metodología de la investigación. México: Ed. Mc Graw Hill. Service, M. V. (20 de enero de 2014). Obtenido de Validate by URI: https://validator.w3.org/

SEO, I. (23 de diciembre de 2014). Posicionamiento Web.Cat. Obtenido de Posicionamiento Web.Cat: https://posicionamientoweb.cat/historiaevolucion-del-posicionamiento-web-seo/

Solis, A. (10 de mayo de 2011). aleyda solis. Obtenido de aleyda solis: https://www.aleydasolis.com/seo/moviles-posicionamiento-buscadores/

Tejada, V. (25 de julio de 2016). Vanessa Tejada. Obtenido de Vanessa Tejada: http://vanessatejada.com/sobre-google/importancia-contenido-webmovil-seo/

Teresa, T. d. (27 de febrero de 2015). Deteresa. Obtenido de Deteresa: http://deteresa.com/seo-moviles/

Thomson, W. (3 de mayo de 1883). Lecture to the Institution of Civil Engineers. (Today in Science) Recuperado el 2013 de septiembre de 1, de http://todayinsci.com/K/Kelvin_Lord/Kelvin_Lord.htm

Ti, O. C. (Octubre de 2013). Guia de Referencia: Accesibilidad de Páginas Web. Obtenido de http://www.once.es/new/servicios-especializadosen-discapacidad- visual/accesibilidad/accesibilidad-en Internet/documentos/AccesibilidadWeb2013.pdf

Torres Bojorquez, A. ( de ). Como buscar información en Internet. (Instituto Tecnológico de Sonora) Recuperado el Junio de 2014, de http://biblioteca.itson.mx/oa/educacion/oa34/como_buscar_informacion_ en_inter net/index.ht

S.L., E. C. (1 de marzo de 2018). Un Como. Obtenido de Un Como: 
https://tecnologia.uncomo.com/articulo/como-usar-pagespeed-insightsde-google-23510.html

Universal, F. S.-A. (15 de diciembre de 2009). Web Content Accessibility $\begin{array}{llll}\text { Guidelines } & \text { (WCAG) } & \text { 2.0. Obtenido de }\end{array}$ http://www.sidar.org/traducciones/wcag20/es/

Vega, Jose A. (2003) La evaluación de la calidad de la información web: aportaciones teóricas y experiencias prácticas Publicado en Recursos informativos: creación, descripción y evaluación. Mérida: Junta de Extremadura, 2003, $\quad$ p. 101-110. (Sociedad de la información, 8).

Tomado de

https://gredos.usal.es/jspui/bitstream/10366/17956/1/DBD_Ev.\%20cal idad\%20inf.

\%20web.pdf

W3C. (2014). Standards. (W3C) Recuperado el 3 de 10 de 2014, de http://www.w3.org/standards/

W3C, U. -V. (20 de enero de 2014). Unicorn - Validador Unificado del W3C. Obtenido de https://validator.w3.org/unicorn/

Web, G. B. (20 de ENERO de 2014). W3C-Guía Breve de Accesibilidad Web. Obtenido de https://www.w3c.es/Divulgacion/GuiasBreves/Accesibilidad 